\title{
Registro Prospectivo de Avaliação da Segurança e Eficácia da Técnica Ulnar na Realização de Procedimentos Coronários
}

\author{
Pedro Beraldo de Andrade ${ }^{1,2}$, Marden André Tebet ${ }^{1,2}$, Mônica Vieira Athanazio de Andrade', \\ Luiz Alberto Mattos ${ }^{1,3}$, André Labrunie ${ }^{1,2}$
}

\section{RESUMO}

Introdução: O uso da técnica radial na realização de procedimentos coronários reduz a incidência de complicações vasculares no sítio de punção, os custos e o tempo de permanência hospitalar, além de propiciar maior conforto ao paciente. No entanto, seu emprego encontra-se prejudicado em $5 \%$ a $15 \%$ dos casos por variações anatômicas da artéria radial, vasoespasmo e teste de Allen negativo. O objetivo do trabalho é avaliar a segurança e a eficácia do acesso ulnar como via alternativa na realização dos procedimentos coronários. Método: Pacientes submetidos a procedimento coronário pela via ulnar foram incluídos em um registro prospectivo. Foram avaliados: o sucesso da técnica, definido como realização de coronariografia com adequada opacificação arterial e intervenção coronária percutânea (ICP) com estenose residual $<30 \%$, sem necessidade de mudança da via de acesso; os tempos de procedimento e de fluoroscopia; e as complicações intra-hospitalares e aos 30 dias de seguimento. Resultados: Entre maio de 2007 e maio de 2008, 102 procedimentos coronários foram incluídos no registro. A média de idade foi de $61 \pm$ 11 anos, sendo $53 \%$ do sexo feminino e $34 \%$ de diabéticos. A taxa de sucesso foi de $94,5 \%$ e $100 \%$ para coronariografia e ICP, respectivamente. Houve baixa incidência de complicações, incluindo $2 \%$ de oclusão assintomática da artéria ulnar e $1 \%$ de hematoma, sem necessidade de reparo vascular ou transfusão sanguínea. Conclusão: $O$ acesso ulnar mostrou-se seguro e eficaz na realização de procedimentos coronários, com elevada taxa de sucesso e virtualmente isento de complicações no sítio de punção. Tratase de uma via de acesso alternativa em serviços que utilizam a técnica radial rotineiramente.

DESCRITORES: Artéria ulnar. Cateterismo cardíaco/métodos. Angioplastia transluminal percutânea coronária/métodos. Angiografia coronária/métodos.

\section{SUMMARY}

Evaluation of Safety and Efficacy of the Transulnar Access for Coronary Procedures

Background: The use of the transradial approach is associated with low rates of complications at the puncture site, reduced costs, shorter hospital stay and is more confortable to the patient. However, its is not feasible in $5 \%$ $15 \%$ of the cases due to significant anatomic variations, vessel spasms and negative results in the Allen's test. The aim of this study was to evaluate the safety and efficacy of the transulnar access as an alternative approach in these situations. Methods: Coronary procedures performed through the transulnar approach were included in a prospective registry. We evaluated the success rates, defined as adequate coronary opacity in coronary angiography or a percutaneous coronary intervention $(\mathrm{PCI})$ with residual stenosis $<30 \%$, with no need to change the access site; procedure and fluoroscopy times and the rate of in-hospital and 30-day complications. Results: Between May 2007 and May 2008, 102 coronary procedures were included in the registry. The mean age was $61 \pm 11$ years, $53 \%$ were female patients and $34 \%$ were diabetic. Success rates were $94.5 \%$ and $100 \%$ for coronary angiography and $\mathrm{PCl}$, respectively. There was a low rate of complications, including $2 \%$ of asymptomatic occlusion of the ulnar artery and $1 \%$ of hematoma not requiring vascular repair or blood transfusion. Conclusions: The use of transulnar access in coronary procedures is safe and efficient, with high success rates and virtually no complications related to the puncture site. It is a good alternative for those experienced with the transradial approach.

DESCRIPTORS: Ulnar artery. Heart catheterization/methods. Percutaneous transluminal coronary angiography/methods. Coronary angiography/methods.
1 Irmandade da Santa Casa de Misericórdia de Marília - Marília, SP

2 Hospital do Coração de Londrina - Londrina, PR.

3 Instituto Dante Pazzanese de Cardiologia - São Paulo, SP.

Correspondência: Pedro Beraldo de Andrade. Praça Athos Fragata,

25/1.101 - Fragata - Marília, SP - CEP 17501-220

E-mail: pedroberaldo@gmail.com

Recebido em: 19/6/2008 • Aceito em: 22/8/2008
A pós sua introdução para realização de procedimentos coronários diagnósticos, em 1989, por Campeau ${ }^{1}$, e terapêuticos, em 1993, por Kiemeneij e Laarman ${ }^{2}$, o acesso radial estabeleceu-se como via segura e custo-efetiva quando comparada às técnicas femoral e braquial. Embora tecnicamente mais desafi- 
adora, como demonstrado por sua curva de aprendizagem $^{3,4}$, associa-se a significativa redução das taxas de sangramento e complicações relacionadas ao sítio de punção $0^{5,6}$. Além disso, propicia maior conforto ao paciente no período de recuperação, deambulação e alta precoce, com conseqüente redução dos custos hospitalares ${ }^{7}$.

Entretanto, em aproximadamente $5 \%$ a $15 \%$ dos casos, suprimento sanguíneo inadequado pelo arco palmar superficial, manifestado pelo teste de Allen modificado negativo, variações anatômicas da artéria radial e ocorrência de vasoespasmo constituem fatores limitantes a seu emprego e causas de falha na obtenção dessa via de acesso ${ }^{8-10}$. Recentemente, a via ulnar vem se apresentando como uma alternativa viável à realização de procedimentos coronários em situações em que o acesso radial se encontra indisponível ${ }^{11}$. Reportamos aqui os resultados de um registro prospectivo de avaliação da segurança e da eficácia da técnica ulnar em pacientes selecionados submetidos a procedimentos coronários diagnósticos e terapêuticos.

\section{MÉTODO}

Em um serviço em que a utilização do acesso radial se situa em torno de $90 \%$ dos casos, pacientes encaminhados para realização de procedimentos coronários que utilizaram a via ulnar, pela impossibilidade de uso do primeiro, foram incluídos em um registro prospectivo de segurança e eficácia.

A eficácia da técnica foi avaliada pelas taxas de sucesso do procedimento, definido como realização de cinecoronariografia e ventriculografia esquerda com adequada opacificação coronária, e intervenção coronária percutânea (ICP), obtendo-se lesão residual inferior a $30 \%$, sem necessidade de mudança da via de acesso. Os tempos de procedimento e de fluoroscopia foram obtidos a partir do início da punção arterial até a retirada do introdutor. Sua segurança foi avaliada pela ocorrência de complicações relacionadas ao sítio de punção, intra-hospitalares e aos trinta dias de seguimento, dados estes obtidos por meio de contato telefônico. Eventos adversos maiores, dentre eles morte, infarto agudo do miocárdio (IAM), cirurgia de revascularização miocárdica (CABG) de urgência e acidente vascular encefálico (AVE), foram contabilizados até o momento da alta hospitalar.

Por meio de hiperextensão do punho e infiltração de 1-2 $\mathrm{ml}$ de xilocaína a $2 \%$, a artéria ulnar foi puncionada 1-3 cm proximal ao osso pisiforme, utilizando-se agulha com cateter de polietileno tipo Jelco ${ }^{\circledR}$ calibre 20 e técnica de Seldinger. Após a punção, foi introduzido um fio-guia de 0,021 polegada (Terumo Corporation, Tokyo, Japão), seguido de pequena incisão cutânea com lâmina de bisturi no 11 e inserção de introdutor curto $(10 \mathrm{~cm}) 5$ ou 6 French (Terumo Corporation, Tokyo, Japão) (Figura 1). Administrava-se uma solução contendo 5.000 UI de sulfato de heparina e $10 \mathrm{mg}$ de mononitrato de isossorbida através da extensão do introdutor. Ao término do procedimento, o introdutor era imediatamente removido e hemostasia realizada com curativo compressivo através de bandagem elástica adesiva porosa (Tensoplast ${ }^{\circledR}$ ) (Figura 2). Procedia-se ao exame clínico do sítio de punção e à avaliação do pulso ulnar no momento da alta, cerca de três a quatro horas após a realização de procedimentos diagnósticos e na manhã seguinte à realização de procedimentos terapêuticos.

\section{RESULTADOS}

Entre maio de 2007 e maio de 2008, foram realizados 102 procedimentos em 90 pacientes, utilizandose o acesso ulnar, sendo 72 coronariografias, 3 estudos pós-revascularização miocárdica, 1 cateterismo cardíaco direito e esquerdo (através de acesso venoso periférico pela veia cefálica), 12 ICPs eletivas e 7 coronariografias seguidas de ICP (ad hoc). As características clínicas estão expressas na Tabela 1. A média de idade foi de $60,8 \pm 11,5$ anos, sendo $53 \%$ dos pacientes do sexo feminino e $34 \%$, diabéticos.

A opção pela utilização da via ulnar decorreu de: presença de pulso ulnar mais amplo e palpável, compa-

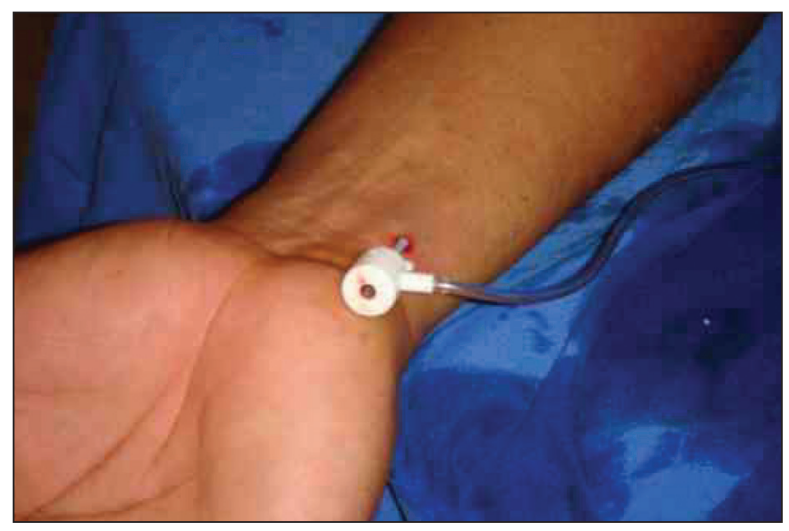

Figura 1 - Introdutor curto 5F em artéria ulnar direita.

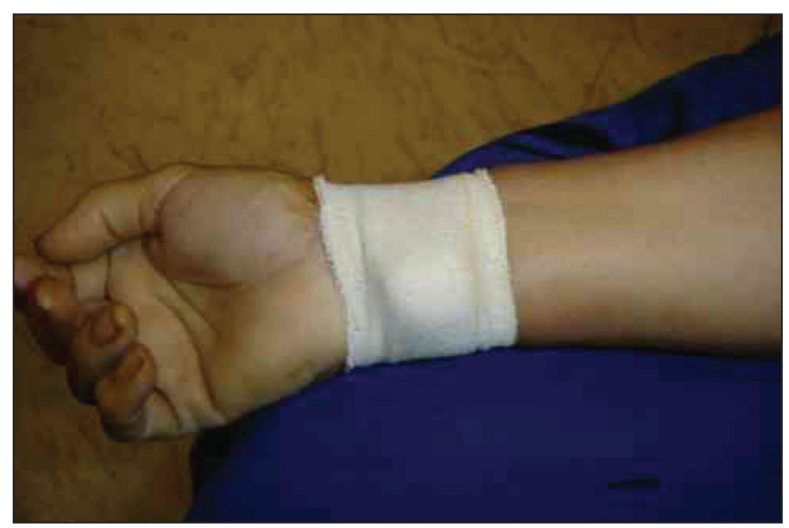

Figura 2 - Curativo compressivo com Tensoplast ${ }^{\circledR}$. 
rativamente ao radial, em 79\% dos casos, buscandose assim evitar a ocorrência de vasoespasmo e desconforto para o paciente; pulso radial ausente, com presença de pulso ulnar e teste de Allen inverso positivo em 12\%; ocorrência de espasmo da artéria radial em $7 \%$; e tortuosidade extrema da artéria radial em $2 \%$, não sendo possível a progressão do fio-guia. A artéria ulnar direita foi utilizada em 92\% dos procedimentos. Para a realização de coronariografia, predominou o uso de cateteres de Judkins direito e esquerdo diâmetro 5 French (89\%), enquanto em ICP utilizaram-se cateteres diâmetro 6 French com lúmen interno de 0,070 polegada e implante de stent em $100 \%$ dos casos.

As taxas de sucesso do procedimento foram de $94,5 \%$ em coronariografias e de $100 \%$ em ICP. Em 2 $(2,7 \%)$ casos houve falha na obtenção do acesso; em 1 caso não foi possível realizar a ventriculografia esquerda em decorrência de espasmo importante da artéria ulnar, porém sem repercussão clínica ou necessidade de anestesia geral; e em outro, embora o acesso ulnar direito tenha sido obtido sem dificuldade, a artéria encontrava-se ocluída em seu terço proximal, sendo convertido o procedimento para via ulnar esquerda. Os tempos de procedimento e de fluoroscopia estão expressos na Tabela 2.

Quanto à ocorrência de complicações relacionadas ao sítio de punção, obtivemos espasmo moderado a grave em 4 (4\%) procedimentos, hematoma em 1 ( $1 \%)$, sem necessidade de cirurgia vascular ou transfusão sanguínea, e oclusão assintomática da artéria ulnar em 2 (2\%) (Tabela 3). Não houve casos de IAM, CABG ou AVE. Houve 2 óbitos hospitalares, em decorrência

\section{TABELA 1}

\section{Características demográficas basais}

\begin{tabular}{lc} 
Variáveis & $\mathbf{n = 9 0}$ \\
\hline Sexo feminino,\% & 53 \\
Média de idade, anos & $60,8 \pm 11,5$ \\
Diabetes melito,\% & 34 \\
Hipertensão arterial sistêmica,\% & 84 \\
Tabagismo,\% & 23 \\
Dislipidemia,\% & 57 \\
Infarto do miocárdio,\% & 17 \\
ICP prévia,\% & 14 \\
Cirurgia de revascularização prévia, \% & 4 \\
Coronariografia prévia,\% & 44 \\
Braquial,\% & 22 \\
Femoral,\% & 18 \\
Radial,\% & 52 \\
$\quad$ Ulnar,\% & 8 \\
\hline ICP = intervenção coronária percutânea; n = número de \\
pacientes.
\end{tabular}

de edema agudo de pulmão pós-procedimento em paciente octogenária com IAM anterior Killip III, e por encefalopatia anóxica pós-parada cardiorrespiratória em paciente de 42 anos, como conseqüência de IAM anterior. Contato telefônico aos trinta dias foi realizado em 98\% dos casos, sem queixas sugestivas de complicações vasculares ou isquêmicas adicionais relacionadas ao sítio de punção.

\section{DISCUSSÃO}

Desde o primeiro relato do uso do acesso ulnar na realização de procedimentos coronários por

TABELA 2

Tempos de procedimento e de fluoroscopia

\begin{tabular}{lcc}
\hline & $\begin{array}{c}\text { Tempo de } \\
\text { procedimento, } \\
\text { min }\end{array}$ & $\begin{array}{c}\text { Tempo de } \\
\text { fluoroscopia, } \\
\text { min }\end{array}$ \\
\hline Coronariografia $(\mathrm{N}=72)$ & $21 \pm 6$ & $3 \pm 2$ \\
ICP eletiva $(\mathrm{N}=12)$ & $39 \pm 16$ & $9 \pm 6$ \\
$\mathrm{ICP}$ ad hoc $(\mathrm{N}=7)$ & $52 \pm 30$ & $16 \pm 14$ \\
\hline ICP = intervenção coronária percutânea; $\mathrm{N}=$ número de \\
procedimentos.
\end{tabular}

TABELA 3

Características dos procedimentos e complicações da via de acesso

\begin{tabular}{|c|c|}
\hline Variáveis & $N=102$ \\
\hline \multicolumn{2}{|l|}{ Indicação do procedimento } \\
\hline Isquemia silenciosa, $\%$ & 19 \\
\hline Angina estável,\% & 42 \\
\hline Síndrome coronária sem supra ST,\% & 35 \\
\hline Infarto agudo do miocárdio,\% & 4 \\
\hline \multicolumn{2}{|l|}{$\begin{array}{l}\text { Número de cateteres utilizados, } \\
\text { média } \pm \text { desvio padrão }\end{array}$} \\
\hline Coronariografia & $2,2 \pm 0,5$ \\
\hline $\mathrm{ICP}$ & 1 \\
\hline Reação vagal,\% & 2 \\
\hline \multicolumn{2}{|l|}{ Espasmo,\% } \\
\hline Leve & 10 \\
\hline Moderado/grave & 4 \\
\hline Hematoma, \% & 1 \\
\hline Sangramento maior, $\%$ & 0 \\
\hline Lesão do nervo ulnar,\% & 0 \\
\hline Oclusão assintomática da artéria ulnar,\% & 2 \\
\hline Pseudo-aneurisma, $\%$ & 0 \\
\hline Fístula arteriovenosa, $\%$ & 0 \\
\hline
\end{tabular}


Terashima et al. ${ }^{12}$, em 2001, em que 7 de 9 pacientes foram submetidos a coronariografia pela técnica ulnar esquerda sem complicações, publicações envolvendo relatos de séries de casos com número variável de pacientes demonstram tratar-se de uma via de acesso alternativa segura e eficaz ${ }^{13}$. Inicialmente utilizada apenas em coronariografias, foi posteriormente avaliada em 128 ICPs eletivas ${ }^{14-17}$ e em 13 casos de ICP primária ${ }^{18}$ e comparada à via transradial em um estudo randomizado envolvendo 431 pacientes submetidos a $I C P$, sem diferenças quanto às complicações na via de acesso e ao sucesso do procedimento ${ }^{19}$.

A taxa de sucesso dos procedimentos realizados pelo acesso ulnar, descrita na literatura, situa-se em torno de $90,9 \%{ }^{11}$. No entanto, os estudos em sua maioria envolvem pacientes selecionados, que apresentam pulso ulnar favorável à execução do procedimento. Em nossa casuística, a taxa de sucesso foi elevada: $94,5 \%$ e $100 \%$ dos procedimentos diagnósticos e terapêuticos, respectivamente. Vassilev et al. ${ }^{20}$, avaliando pacientes consecutivos não selecionados, reportaram taxa de sucesso de apenas 45\%. De 131 pacientes, 29 não apresentavam pulso ulnar palpável e em 33 não foi possível concluir o procedimento, sendo a razão principal a falha na obtenção da via de acesso. Aptecar et al. ${ }^{19}$, entretanto, obtiveram taxa de sucesso de $90,5 \%$ na realização de procedimentos pela via transulnar em pacientes não-selecionados em estudo randomizado.

Embora operadores experientes e habituados ao uso da técnica radial não encontrem dificuldades na utilização da via ulnar, verifica-se uma curva de aprendizagem, demonstrada pela redução do número de tentativas de punção para obtenção do $\operatorname{acesso}^{16}$. A despeito disso, não observamos em nosso registro maiores tempos de procedimento e de fluoroscopia comparados a coortes históricas de pacientes submetidos a procedimentos terapêuticos eletivos ou ad hoc pela via radial ${ }^{5,21,22}$.

As baixas taxas de complicações relacionadas ao sítio de punção observadas em nosso estudo estão de acordo com os achados da literatura ${ }^{11}$, corroborando a premissa de que as vantagens obtidas com a utilização do acesso radial seriam alcançadas pela via ulnar. Nossa população reflete uma prática clínica do mundo real, sem critérios clínicos de exclusão. Aproximadamente $40 \%$ dos pacientes apresentavam síndrome coronária aguda e encontravam-se em regime antitrombótico agressivo, incluindo terapia antiplaquetária, heparinização e inibidores dos receptores da glicoproteína $\mathrm{Ilb} / \mathrm{Illa}^{23}$. Foi observado apenas um caso de hematoma importante, envolvendo o antebraço, em paciente de 79 anos que havia utilizado estreptoquinase nas cinco horas que antecederam o procedimento, porém sem necessidade de reparo cirúrgico vascular ou transfusão sanguínea.
Apesar da proximidade da artéria ulnar com o nervo e a veia correspondentes, não houve complicação neurológica por punção inadvertida ou compressão local por hematoma. Foi infreqüente a ocorrência de oclusão assintomática da artéria ulnar $(2 \%)$, sem repercussão isquêmica da mão. A presença de arco palmar profundo completo proveniente da artéria radial em aproximadamente $95 \%$ da população ${ }^{24}$, bem como a grande capacidade de reserva funcional da circulação da mão, representada pela circulação colateral ${ }^{25,26}$, constituem fatores protetores contra a ocorrência de complicações isquêmicas e justificariam a realização do procedimento na vigência de pulso radial ausente, mas com teste de Allen inverso positivo, que se situou em $12 \%$ das indicações em nossa casuística.

São limitações do estudo o fato de se tratar de um registro prospectivo, não-randomizado, envolvendo número relativamente pequeno de pacientes. A ausência de seguimento com exame de imagem, como ultra-som Doppler, pode subestimar a real porcentagem de complicações, como oclusões arteriais assintomáticas, fístula arteriovenosa e pseudo-aneurisma. A utilização de materiais reprocessados pode ter influenciado negativamente a ocorrência de espasmo e o tempo de procedimento.

\section{CONCLUSÃO}

Em serviços que utilizam a técnica radial rotineiramente, o acesso ulnar surge como uma alternativa segura, viável e eficaz à realização de procedimentos coronários diagnósticos e terapêuticos, evitando-se sua conversão para a técnica transfemoral, sobretudo em situações como variações anatômicas da artéria radial, vasoespasmo e negatividade do teste de Allen modificado.

\section{REFERÊNCIAS BIBLIOGRÁFICAS}

1. Campeau L. Percutaneous radial artery approach for coronary angiography. Cathet Cardiovasc Diagn. 1989;16(1):3-7.

2. Kiemeneij F, Laarman GJ. Percutaneous transradial artery approach for coronary stent implantation. Cathet Cardiovasc Diagn. 1993;30(2):173-8.

3. Louvard Y, Lefevre T, Morice MC. Radial approach: what about the learning curve? Cathet Cardiovasc Diagn. 1997;42(4):467-8.

4. Labrunie A, Silveira W, Contero L, mais 3 autores, et al. Transradial approach to coronary angiography: the reality of the learning curve evaluated in a comparative, randomized, multicenter study. Am J Cardiol. 2001;88(5 Suppl. 1):111G-112G.

5. Kiemeneij F, Laarman GJ, Odekerken D, Slagboom T, van der Wieken R. A randomized comparison of percutaneous transluminal coronary angioplasty by the radial, brachial and femoral approaches: the access study. J Am Coll Cardiol. $1997 ; 29(6): 1269-75$.

6. Louvard Y, Lefèvre T, Allain A, Morice M. Coronary angiography through the radial or the femoral approach: The CARAFE study. Catheter Cardiovasc Interv. 2001;52(2):181-7.

7. Cooper CJ, El-Shiekh RA, Cohen DJ, Blaesing L, Burket MW, Basu A, et al. Effect of transradial access on quality of life and cost of cardiac catheterization: A randomized comparison. Am Heart J. 1999;138(3 Pt 1):430-6.

8. Benit E, Vranckx P, Jaspers L, Jackmaert R, Poelmans C, 
Andrade PB, et al. Registro Prospectivo de Avaliação da Segurança e Eficácia da Técnica Ulnar na Realização de Procedimentos Coronários. Rev Bras Cardiol Invas. 2008;16(3):312-316.

Coninx R. Frequency of a positive modified Allen's test in 1,000 consecutive patients undergoing cardiac catheterization. Cathet Cardiovasc Diagn. 1996;38(4):352-4.

9. Yokoyama N, Takeshita S, Ochiai M, Koyama Y, Hoshino $\mathrm{S}$, Isshiki $\mathrm{T}$, et al. Anatomic variations of the radial artery in patients undergoing transradial coronary intervention. Catheter Cardiovasc Interv. 2000;49(4):357-62.

10. Valsecchi O, Vassileva A, Musumeci G, Rossini R, Tespili M, Guagliumi G, et al. Failure of transradial approach during coronary interventions: anatomic considerations. Catheter Cardiovasc Interv. 2006;67(6):870-8.

11. Roberts EB, Palmer N, Perry RA. Transulnar access for coronary angiography and intervention: an early review to guide research and clinical practice. J Invasive Cardiol. $2007 ; 19(2): 83-7$.

12. Terashima $M$, Meguro $T$, Takeda $H$, Endoh $N$, Ito $Y$, Mitsuoka $M$, et al. Percutaneous ulnar artery approach for coronary angiography: a preliminary report in nine patients. Catheter Cardiovasc Interv. 2001;53(3):410-4.

13. Roberts EB, Palmer N, Perry RA. Transulnar access for coronary angiography and intervention: an early review to guide research and clinical practice. I Invasive Cardiol. $2007 ; 19(2): 83-7$.

14. Dashkoff N, Dashkoff PB, Zizzi JA Sr, Wadhwani J, Zizzi JA Jr. Ulnar artery cannulation for coronary angiography and percutaneous coronary intervention: case reports and anatomic considerations. Catheter Cardiovasc Interv. 2002;55(1):93-6.

15. Mangin L, Bertrand OF, De La Rochellière R, Proulx G, Lemay $\mathrm{R}$, Barbeau G, et al. The transulnar approach for coronary intervention: a safe alternative to transradial approach in selected patients. J Invasive Cardiol. 2005;17(2):77-9.

16. Aptecar E, Dupouy P, Chabane-Chaouch M, Bussy N, Catarino G, Shahmir A, et al. Percutaneous transulnar artery approach for diagnostic and therapeutic coronary intervention. J Invasive Cardiol. 2005;17(6):312-7.
17. Rath PC, Purohit BV, Navasundi GB, Sitaram, Reddy AM. Coronary angiogram and intervention through transulnar approach. Indian Heart J. 2005;57(4):324-6.

18. Limbruno U, Rossini R, De Carlo M, Amoroso G, Ciabatti $\mathrm{N}$, Petronio AS, et al. Percutaneous ulnar artery approach for primary coronary angioplasty: safety and feasibility. Catheter Cardiovasc Interv. 2004;61(1):56-9.

19. Aptecar E, Pernes JM, Chabane-Chaouch M, Bussy N, Catarino G, Shahmir A, et al. Transulnar versus transradial artery approach for coronary angioplasty: the PCVI-CUBA study. Catheter Cardiovasc Interv. 2006;67(5):711-20.

20. Vassilev D, Smilkova D, Gil R. Ulnar artery as access site for cardiac catheterization: anatomical considerations. J Interv Cardiol. 2008;21(1):56-60.

21. Louvard $Y$, Ludwig J, Lefèvre $T$, Schmeisser $A$, Brück $M$, Scheinert $\mathrm{D}$, et al. Transradial approach for coronary angioplasty in the setting of acute myocardial infarction: a dual-center registry. Catheter Cardiovasc Interv. 2002;55(2):206-11.

22. Philippe F, Larrazet F, Meziane T, Dibie A. Comparison of transradial vs. transfemoral approach in the treatment of acute myocardial infarction with primary angioplasty and abciximab. Catheter Cardiovasc Interv. 2004;61(1):67-73.

23. Tebet MA, Andrade PB, Gentile M, Mattos LA, Labrunie A. Angioplastia primária via radial com doses aumentadas de tirofiban: uma técnica segura e efetiva. Rev Bras Cardiol Invas. $2007 ; 15(3): 255-60$.

24. Vogelzang RL. Arteriography of the hand and wrist. Hand Clin. $1991 ; 7(1): 63-86$.

25. Greenwood MJ, Della-Siega AJ, Fretz EB, Kinloch D, Klinke $P$, Mildenberger $R$, et al. Vascular communications of the hand in patients being considered for transradial coronary angiography: is the Allen's test accurate? J Am Coll Cardiol. $2005 ; 46(11): 2013-7$.

26. Lanspa TJ, Reyes AP, Oldemeyer JB, Willians MA. Ulnar artery catheterization with occlusion of corresponding radial artery. Catheter Cardiovasc Interv. 2004;61(2):211-3. 